\title{
Cloned Platelet Thrombin Receptor Is Necessary for Thrombin-induced Platelet Activation
}

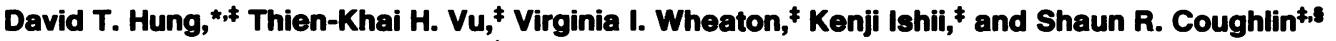 \\ ${ }^{*}$ Department of Laboratory Medicine; ${ }^{\ddagger}$ Cardiovascular Research Institute; and §Department of Medicine, \\ University of California, San Francisco, San Francisco, California 94143
}

\begin{abstract}
Platelet activation by thrombin is critical for hemostasis and thrombosis. Structure-function studies with a recently cloned platelet thrombin receptor suggest that a hirudin-like domain in the receptor's extracellular amino terminal extension is a thrombin-binding determinant important for receptor activation. We now report that a peptide antiserum to this domain is a potent and specific antagonist of thrombin-induced platelet activation. This study demonstrates that the cloned platelet thrombin receptor is necessary for platelet activation by thrombin, and provides a strategy for developing blocking monoclonal antibodies of potential therapeutic value. (J. Clin. Invest. 1992. 89:1350-1353.) Key words: antibody • inhibitor • serine proteases $\bullet$ thrombosis $\bullet$ hirudin
\end{abstract}

\section{Introduction}

Thrombin-induced platelet activation plays a pivotal role in arterial thrombosis $(1,2)$. We recently isolated a cDNA clone encoding a functional platelet thrombin receptor (3). The clone encoded a member of the seven transmembrane span family of receptors with a 100 -residue amino terminal extension. The amino acid sequence of this extracellular extension revealed a thrombin cleavage site (LDPR/S with/representing the point of cleavage) and a "hirudin-like domain" (DKYEPFWEDEE). Structure-function studies with the cloned receptor and receptor-based peptides suggest the following model of receptor activation. Thrombin binds its receptor via at least two sites: thrombin's S1-S4 subsites interact with the receptor's thrombin cleavage site (LDPR/S), while thrombin's anion-binding exosite interacts with the receptor's hirudin-like domain $(4,5)$. Thrombin then cleaves the receptor at the LDPR/S site, unmasking a new receptor amino terminus beginning with the sequence SFLL . . . ; this new amino terminus then functions as a tethered peptide ligand to effect receptor activation (3). A synthetic peptide mimicking this new amino terminus functioned as a full agonist for platelet activation (3), suggesting

Address correspondence and reprint requests to Dr. Shaun R. Coughlin, University of California, San Francisco, Cardiovascular Research Institute, Box 0524, HSW 846, San Francisco, CA 94143.

Received for publication 30 October 1991 and in revised form 5 December 1991.

J. Clin. Invest.

(c) The American Society for Clinical Investigation, Inc. 0021-9738/92/04/1350/04 \$2.00

Volume 89, April 1992, 1350-1353 that activation of the cloned receptor or a highly related receptor was sufficient for platelet activation.

Is the cloned platelet thrombin receptor necessary for platelet activation by thrombin? We now report that a peptide antiserum to the receptor's hirudin-like domain specifically blocks thrombin-induced responses in platelets. These studies show that activation of the cloned thrombin receptor is indeed necessary for platelet activation by thrombin, and buttress the importance of the receptor's hirudin-like domain in thrombin-receptor interactions. Moreover, these studies establish the cloned thrombin receptor as a potential target for antiplatelet therapy, and provide a strategy for the development of blocking monoclonal antibodies of potential therapeutic value.

\section{Methods}

Materials. Purified human $\alpha$-thrombin was a generous gift from Dr. John W. Fenton II, Albany Medical College of Union University, Albany, NY. Thrombin receptor agonist peptide SFLLRNPNDKYEPF, "scrambled" peptide FSLLRNPNDKYEPF, and peptides used for antigen or controls (YEPFWEDEEKNESGLTEY ["360"], YEPFWEDEEKNESGLTEYC [“360-1"], PESKATNATLDPRSFLL [“359”], and PESKATNATLDPRSFLLC ["359-1"]) were synthesized by UCSF's Biomolecular Resource Center (see Fig. $1 A$ ). Rabbit polyclonal antisera were raised to peptides $359-1$ and 360-1 conjugated by maleido-bis-succinimide to keyhole limpet hemocyanin (KLH) (6). Antisera were screened initially by ELISA against antigen and irrelevant peptides (6), then by immunoblot (see Fig. $1 B$ ). All platelet and oocyte experiments used IgG purified from immune or preimmune sera, using protein-A agarose (Pierce Chemical Co., Rockford, IL) and dialysis against phosphate-buffered saline. Stably transfected Rat 1 cells expressing the cloned human platelet thrombin receptor were prepared by standard techniques (7). Antiserum raised to KLH-conjugated peptide 360-1 is referred to as "hirudin-like domain antiserum." IgG purified from this antiserum is referred to as "hirudin-like domain IgG." Preimmune sera and "irrelevant" peptide antiserum raised to monocyte chemoattractant protein were used as controls.

Platelet aggregation and ATP secretion studies. Washed platelets were prepared as previously described (8), and unless otherwise noted, were suspended in modified Tyrode's buffer, $\mathrm{pH} 7.4$ with $2 \mathrm{mM}$ magnesium and $1 \mathrm{mM}$ calcium at a concentration of $\sim 3 \times 10^{8}$ platelets $/ \mathrm{ml}$. All platelet studies were performed in a total volume of $500 \mu$, with 20 $\mu$ l Chromolume ${ }^{\oplus}$ reagent (Chrono-log Corp., Havertown, PA). Platelet ATP secretion and aggregation were quantitated independently by measuring changes in luminescence and light transmittance, respectively, in a dual-channel lumiaggregometer (Chrono-log Corp.) (9). Stirred platelets were used to ensure rapid and uniform distribution of agonist, and initial rates of secretion were determined as defined in the appropriate figure legends.

Xenopus oocyte studies. Xenopus oocytes were harvested from female Xenopus laevis and processed using published techniques (10, 11). Individual oocytes were microinjected with cloned functional 
platelet thrombin receptor cRNA and cultured for $48 \mathrm{~h}$ at $18^{\circ} \mathrm{C}$ in modified Barth's solution (3). Thrombin- or agonist peptide-induced calcium mobilization was measured by ${ }^{45} \mathrm{Ca}$ release from receptor-expressing oocytes $(3,11)$.

\section{Results and Discussion}

The importance of the thrombin receptor's hirudin-like domain in thrombin-receptor interaction $(4,5)$ suggested that an antiserum to this domain might block receptor activation by thrombin. Accordingly, we raised rabbit antiserum to a peptide representing this domain; IgG purified from this antiserum was used for these studies.

The hirudin-like domain antiserum recognized a broad band at $\sim 70 \mathrm{kD}$ in immunoblots of lysate from mammalian cells transfected with thrombin receptor cDNA (Fig. $1 B$ ). The relative molecular mass predicted for the unglycosylated thrombin receptor is $\sim 44 \mathrm{kD}$. The pattern of molecular weights seen presumably reflects receptor glycosylation and/or aberrant migration of this highly hydrophobic protein. A similar pattern was recognized in lysates from human platelets. No signal was detected in lysates from the untransfected parent cell line. These data suggested that the antiserum specifically recognized the thrombin receptor protein expressed by the transfected cells and platelets.

A

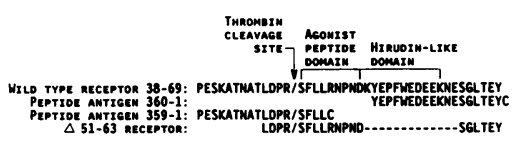

B

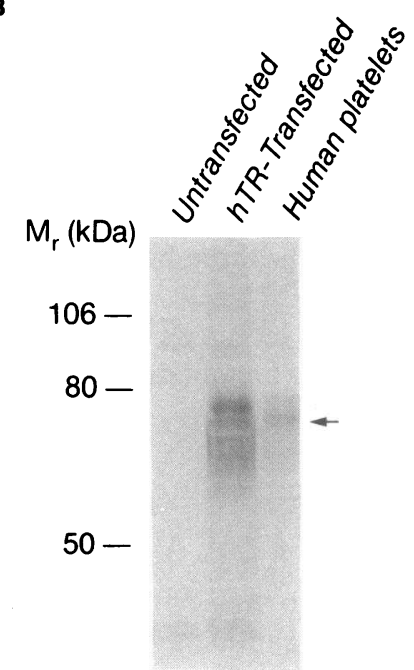

Figure 1. Characterization of the thrombin receptor hirudin-like domain antiserum. $(A)$ Amino acid sequences of the native thrombin receptor's amino terminal extension with its hirudin-like domain, of peptides to which antisera were raised, and of the hirudin-like domain deletion mutant thrombin receptor (dashes indicate deleted residues). $(B)$ Immunoblot of lysates from untransfected (left lane), receptortransfected (central lane), and human platelets (right lane). Approximately $10^{6}$ untransfected Rat 1 cells, Rat 1 cells stably transfected with the platelet thrombin receptor

cDNA clone, and washed human platelets (prepared from $3 \mathrm{~cm}^{3}$ of blood) were lysed in $100 \mu \mathrm{l}$ phosphate-buffered saline, calcium and magnesium-free, with $0.04 \%$ EDTA, $1 \mathrm{mM}$ APMSF, $1 \mathrm{mM}$ benzamidine, $1 \mathrm{mM}$ leupeptin, $1 \mathrm{mM}$ aprotinin, and $1 \%$ Triton $\mathrm{X}-100$ for $15 \mathrm{~min}$ at $4^{\circ} \mathrm{C}$. The lysates were clarified by centrifugation at 14,000 rpm for $5 \mathrm{~min}$, and supernatants were analyzed by $9 \%$ SDS-PAGE gel electrophoresis under nonreducing conditions. After transfer to nitrocellulose, the immunoblot was blocked with $5 \%$ powdered milk for $1 \mathrm{~h}$ at room temperature, and then incubated with $1.5 \mu \mathrm{g} / \mathrm{ml}$ of the hirudin-like domain IgG overnight at $4^{\circ} \mathrm{C}$. Primary antibody was detected with goat anti-rabbit IgG conjugated to horseradish peroxidase (6)
The hirudin-like domain antiserum effectively blocked thrombin-induced platelet activation. $30 \mu \mathrm{g} / \mathrm{ml} \mathrm{IgG} \mathrm{purified}$ from this antiserum completely inhibited platelet ATP secretion and aggregation in response to $1 \mathrm{nM}$ thrombin (Fig. 2, $A$ and $B$ ). This concentration of thrombin typically elicits $\mathrm{EC}_{50}$ or greater platelet responses. IgG prepared from an irrelevant rabbit antiserum had no inhibitory effects on thrombin-induced platelet activation, and was used as "control IgG" in most experiments (Figs. 2-5). IgG prepared from preimmune serum also had no inhibitory effects (not shown). Furthermore, no platelet agonist activity could be demonstrated in the hirudin-

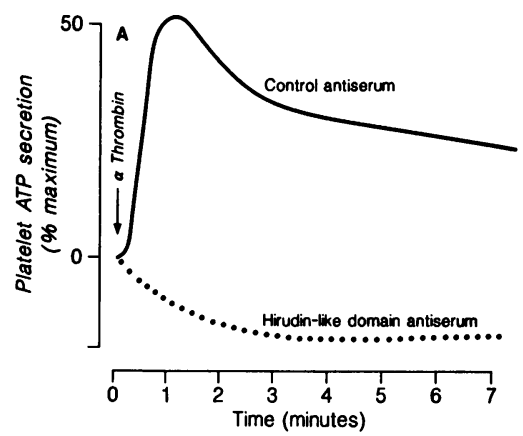

B
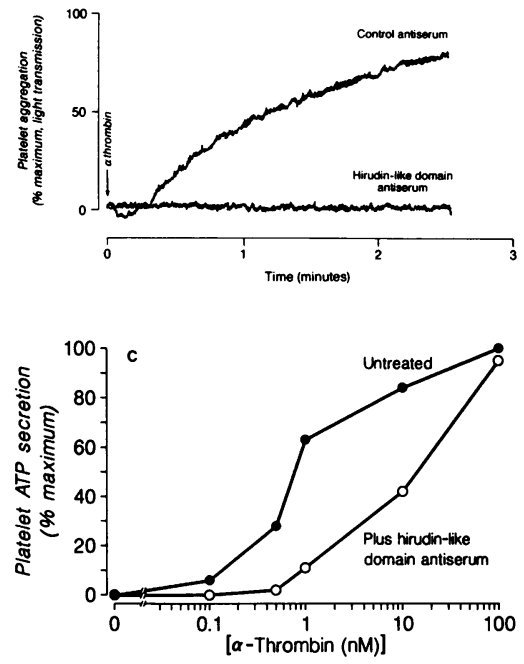

Figure 2. Hirudin-like domain antiserum inhibits thrombin-induced platelet ATP secretion and platelet aggregation. $(A)$

Representative tracing of thrombin-induced platelet ATP secretion curves in the presence of hirudin-like domain (dotted lines) or control (solid lines) IgG. (B) Representative recordings of thrombin-induced platelet aggregation in the presence of hirudin-like domain or control IgG. Curves in $A$ and $B$ are representative of over 20 recordings. (C) Platelet ATP secretion in response to varying concentrations of thrombin in the presence of hirudin-like domain (open circles) or control (closed circles) IgG. $480 \mu$ l of washed platelets (8) suspended in Tyrode's buffer at a concentration of $\sim 3$ $\times 10^{8}$ platelets $/ \mathrm{ml}$ were incubated for $5 \mathrm{~min}$ at

$37^{\circ} \mathrm{C}$ with IgG purified from hirudin-like domain antiserum $(30 \mu \mathrm{g} /$ $\mathrm{ml}$ final concentration), or an equal concentration of IgG from irrelevant antiserum or preimmune antiserum. $10 \mu \mathrm{l}$ of Chromolume ${ }^{\oplus}$ reagent was added, followed by challenge with $1 \mathrm{nM} \alpha$-thrombin. This concentration of thrombin typically gives $\mathrm{EC}_{\mathbf{5 0}}$ or greater platelet responses. Platelet ATP secretion and platelet aggregation were measured in a lumiaggregometer (9). In panel $C$, platelets were pretreated with hirudin-like domain or control IgG and challenged with the concentrations of thrombin indicated, and platelet ATP secretion was measured. Points represent the mean of the peak ATP secretion noted at each thrombin concentration indicated in three replicate determinations. Platelet ATP secretion and aggregation data in all experiments are expressed as a percentage of maximum; $100 \%$ ATP secretion is defined as the peak luminescence signal obtained after the addition of $100 \mathrm{nM} \alpha$-thrombin to control IgG-pretreated platelets; $100 \%$ aggregation is defined as the light transmission obtained 2.5 min after the addition of $100 \mathrm{nM} \alpha$-thrombin to control IgG-pretreated platelets. All experiments were replicated at least three times. 


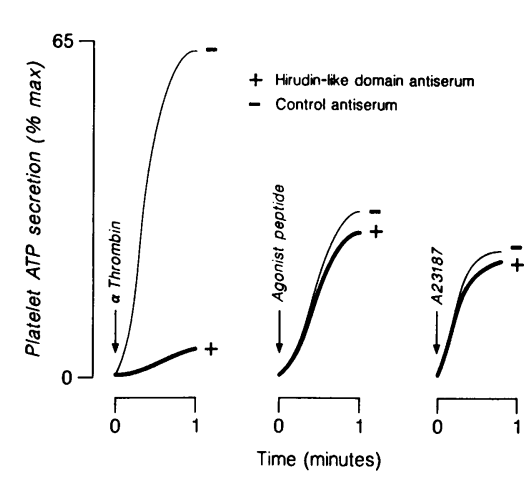

Figure 3. Hirudin-like domain antiserum specifically blocks thrombin-induced platelet ATP secretion. Washed platelets were incubated in the presence of 30 $\mu \mathrm{g} / \mathrm{ml} \mathrm{IgG}$ from hirudin-like domain antiserum $(+)$, or the equivalent final concentration of control IgG (-) and platelet ATP secretion in response to thrombin (1 nM), agonist peptide SFLLRNPNDKYEPF (30 $\mu \mathrm{M})$, and A23187 (60 nM) was measured as described in Fig. 2. 100\% ATP secretion is defined as the peak luminescence signal obtained after the addition of $100 \mathrm{nM} \alpha$-thrombin to control IgG-pretreated platelets. These figures are tracings of platelet ATP secretion curves and are representative of the results obtained in four replicate experiments.

like domain IgG preparation, suggesting that the inhibitory effects of the antiserum on platelet activation by thrombin were not due to platelet desensitization by the antiserum.

The $\mathrm{IC}_{50}$ of the hirudin-like domain IgG to block $\mathrm{EC}_{50}$ concentrations of thrombin for platelet ATP secretion was $\sim 7.5$ $\mu \mathrm{g} / \mathrm{ml}$. This inhibition was competitive, as increasing concentrations of thrombin overcame the inhibitory effects of the antiserum on responses in both the platelet system (Fig. $2 \mathrm{C}$ ) and in oocytes expressing the cloned thrombin receptor (Fig. 5; see below).

The inhibitory effect of the hirudin-like domain IgG was specific for thrombin-induced responses. It blocked platelet $\mathrm{ATP}$ secretion in response to $\mathrm{EC}_{50}$ concentrations of thrombin,

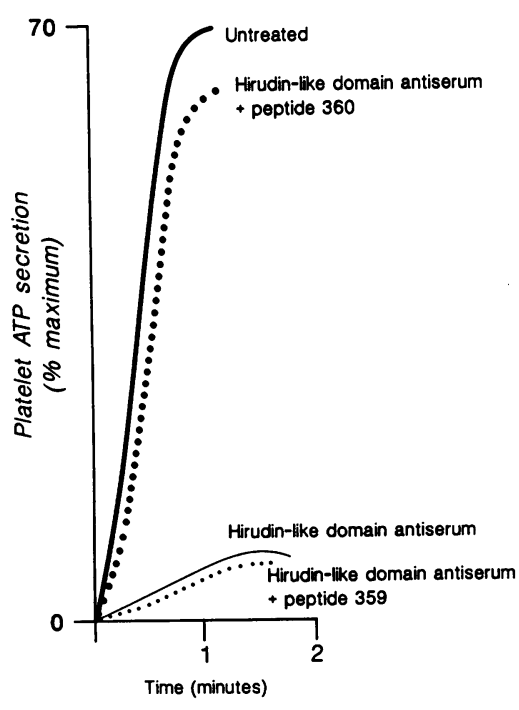

Figure 4. The inhibitory effect of the hirudin-like domain antiserum on thrombin-induced platelet ATP secretion is ablated by preincubation with a peptide representing the hirudinlike domain. Washed platelets were incubated with $30 \mu \mathrm{g} / \mathrm{ml} \mathrm{IgG} \mathrm{from}$ hirudin-like domain antiserum or an equivalent final concentration of control IgG in the presence (dotted lines) or absence (solid lines) of $50 \mu \mathrm{M}$ peptide PESKATNATLDPRSFLL (359), or hirudin-like domain peptide YEPF-

WEDEEKNESGLTEY (360), as described in Fig. 2. Platelets were then challenged with $1 \mathrm{nM} \alpha$-thrombin and platelet ATP secretion was measured. $100 \%$ ATP secretion is defined as the peak luminescence signal obtained after the addition of $100 \mathrm{nM} \alpha$-thrombin to control IgG-pretreated platelets. These figures are tracings of platelet ATP secretion curves and are representative of the results obtained in three replicate experiments.

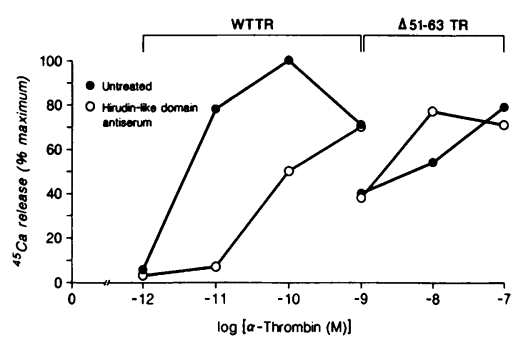

Figure 5. Effects of hirudin-like domain antiserum on thrombin-induced calcium mobilization in Xenopus oocytes expressing wild type thrombin receptor (WTTR) or mutant thrombin receptors lacking the hirudin-like domain ( $\triangle 51-63$ TR).

Oocytes expressing cRNA encoding wild type thrombin receptor (WTTR) or mutant thrombin receptors lacking the hirudin-like domain ( $\triangle 51-63 \mathrm{TR}$ ) were preincubated with $30 \mu \mathrm{g} / \mathrm{ml} \mathrm{IgG} \mathrm{from} \mathrm{hiru-}$ din-like domain antiserum (open circles) or an equivalent final concentration of control IgG (closed circles) for $5 \mathrm{~min}$, and then treated with $\alpha$-thrombin at the concentrations indicated for $10 \mathrm{~min}$ at room temperature. Agonist-induced ${ }^{45} \mathrm{Ca}$ release was measured, as previously described $(3,10)$.

but did not block platelet responses to $\mathrm{EC}_{50}$, or even lower concentrations of thrombin receptor agonist peptide (Fig. 3). Thrombin receptors on antibody-treated platelets are thus available for activation by agonist peptide, which bypasses receptor proteolysis and activates the thrombin receptor directly $(3,4)$. The ability of the hirudin-like domain IgG to block thrombin- but not agonist peptide-induced platelet activation suggests that the hirudin-like domain IgG does act by specifically binding the receptor's hirudin-like domain, thereby blocking thrombin interaction with the receptor's amino terminal extension, but not agonist peptide interaction with the body of the receptor. The specificity of the antiserum is further confirmed by its inability to block platelet activation by sub$\mathrm{EC}_{50}$ concentrations of calcium ionophore (Fig. 3).

The inhibitory effects of the hirudin-like domain IgG preparation were ablated by preincubating the $\mathrm{IgG}$ with the peptide to which it was raised (peptide 360, Fig. 4), but not by preincubation with an unrelated peptide (peptide 359, Fig. 4). These data strongly suggest that the inhibitory activity of this preparation is due to antibodies that recognize the thrombin receptor's hirudin-like domain.

To further test the importance of the receptor's hirudin-like domain in the action of this antiserum, a mutant receptor lacking this domain was expressed in Xenopus oocytes and thrombin responses assessed. Deletion of the receptor's hirudin-like domain caused the concentration response to thrombin to shift rightward, but had no significant effect on the concentration response to peptide (4). Hirudin-like domain $\mathrm{IgG}$ inhibited thrombin-induced calcium mobilization in oocytes expressing the wild-type thrombin receptor, right shifting the concentration response curve by $\sim 30$-fold and toward that seen for deletion mutant receptor ( $\Delta 51-63$; Fig. 5). Moreover, the receptor's hirudin-like domain was indeed required for the inhibition by the hirudin-like domain antiserum, in that the antiserum did not inhibit thrombin responses in oocytes expressing the $\Delta 51-63$ receptor (Fig. 5).

Similar studies with multiple different antisera to various receptor domains support the role of the cloned receptor in thrombin-induced platelet activation (not shown). IgG from three different rabbit antisera raised to the receptor's hirudinlike domain peptide (peptide 360-1, Fig. $1 A$ ) all blocked thrombin-induced platelet activation. Additional antisera were 
raised to other receptor-based peptides. Like the hirudin-like domain antiserum, all recognized the receptor by immunoblot. Three of these antisera were raised to a peptide containing the LDPR/SFLL sequence which represents the receptor's thrombin cleavage site and a portion of its agonist peptide domain (peptide 359-1, Fig. $1 A$ ). IgG from two of these antisera blocked thrombin-induced platelet activation, but less effectively than the hirudin-like domain antisera. Prevention of agonist peptide function or of receptor cleavage by antibody binding at or near this sequence may account for the activity of the inhibitory 359-1 antisera.

IgG from a third antiserum to peptide 359-1 had no inhibitory activity at up to $30 \mu \mathrm{g} / \mathrm{ml}$, nor did IgG from two other antisera raised to a peptide representing the thrombin receptor's second extracellular loop. In the absence of information on the fraction of receptor's occupied by antibody on the intact platelet, these negative data cannot be used to draw conclusions regarding specific structure-function issues; such studies will become possible when receptor monoclonal antibodies are available. These data do suggest, however, that blocking activity may not be a general feature of all receptor antisera.

In summary, these studies strongly demonstrate that an antiserum to the cloned thrombin receptor's hirudin-like domain blocks thrombin-induced platelet activation. Previous reports have suggested that activation of the cloned thrombin receptor is sufficient for platelet activation by showing that thrombin receptor agonist peptide mimics thrombin-induced platelet secretion and aggregation (3) and thrombin-induced phosphoinositide signaling (17). The present study now "closes the loop" on the clone; the demonstration that peptide antisera to the cloned thrombin receptor block thrombin-induced platelet activation now strongly suggests that the cloned receptor is necessary for platelet activation by thrombin. This result does not preclude a role for other thrombin receptors or binding proteins in platelet activation (12), but implies that such receptors would play an adjunctive rather than central role.

Mutation of the thrombin receptor's hirudin-like domain by deletions or by alanine scanning suggested the importance of this domain in receptor activation by thrombin (4). Receptor peptides representing this domain directly bind thrombin, apparently by interacting with thrombin's anion-binding exosite (5). The inhibitory activity of the hirudin-like domain antiserum buttresses these studies by providing functional evidence for the importance of the thrombin receptor's hirudinlike domain in intact platelets.

The effectiveness with which this polyclonal antiserum blocks thrombin-induced platelet activation establishes the cloned thrombin receptor as a potential target for antiplatelet therapy as well as the feasibility of developing antibody-based thrombin receptor antagonists. Blocking monoclonal antibodies to the receptor's hirudin-like domain or other domains will be valuable reagents for establishing the role of thrombin receptor activation in various in vivo processes. Such antibodies may be of therapeutic value as antithrombotic agents or as antagonists of thrombin's inflammatory $(13,14)$, or proliferative actions $(15,16)$.

\section{Acknowledgments}

We thank Dr. John Fenton II (Wadsworth Center/Albany Medical College) for his generous gift of $\alpha$-thrombin, and Dr. Laurence Corash (UCSF) for the use of the lumiaggregometer.

This work was supported in part by the Physician-Scientist Award from the National Institutes of Health HL-0248-01 to D. T. Hung, the Tobacco-Related Disease Research Program of the University of California 2RT19, and NIH HL-44907 and HL43801 to S. R. Coughlin. This work was done during the tenure of an award from the American Heart Association and SmithKline Beecham to S. R. Coughlin.

\section{References}

1. Hanson, S. R., and L. A. Harker. 1988. Interruption of acute platelet-dependent thrombosis by the synthetic antithrombin PPACK. Proc. Natl. Acad. Sci. USA. 85:3184-3188.

2. Eidt, J. F., P. Allison, S. Noble, J. Ashton, P. Golino, J. McNatt, L. M. Buja, and J. T. Willerson. 1989. Thrombin is an important mediator of platelet aggregation in stenosed canine coronary arteries with endothelial injury. J. Clin. Invest. 84:18-27.

3. Vu, T-K. H., D. T. Hung, V. I. Wheaton, and S. R. Coughlin. 1991. Molecular cloning of a functional thrombin receptor reveals a novel proteolytic mechanism of receptor activation. Cell. 64:1057-1068.

4. Vu, T-K. H., V. I. Wheaton, D. T. Hung, I. Charo, and S. R. Coughlin. 1991. Domains specifying thrombin-receptor interaction. Nature (Lond.). 353:674-677.

5. Liu, L-W., T-K. H. Vu, C. T. Esmon, and S. R. Coughlin. 1991. The region of the thrombin receptor resembling hirudin binds to thrombin and alters enzyme specificity. J. Biol. Chem. 266:16 977-16 980.

6. Harlow, E., and D. Lane. 1988. Antibodies: a laboratory manual. Cold Spring Harbor Laboratory, Cold Spring Harbor, NY.

7. Hung, D. T., T. K. H. Vu, N. A. Nelken, and S. R. Coughlin. 1992. Thrombin-induced events in non-platelet cells are mediated by the unique proteolytic mechanism established for the cloned platelet thrombin receptor. J. Cell Biol. 116:827-832.

8. Baenzinger, N. G., and P. W. Majerus. 1974. Isolation of human platelets and platelet surface membranes. Methods Enzymol. 31:149-155.

9. Charo, I. F., R. D. Feinman, and T. C. Detwiler. 1977. Interrelations of platelet aggregation and secretion. J. Clin. Invest. 63:866-873.

10. Coleman, A. 1984. Translation of eukaryotic mRNA in Xenopus oocytes. In Transcription and Translation: A practical approach. B. D. Hames and S. J. Higgins, editors. IRL Press, Oxford. pp. 271-302.

11. Williams, J. A., D. J. McChesney, M. C. Calayag, V. R. Lingappa, and C. D. Logsdon. 1988. Expression of receptors for cholecystokinin and other $\mathrm{Ca}^{2+}$ mobilizing hormones in Xenopus oocytes. Proc. Natl. Acad. Sci. USA. 85:49394943.

12. Okumura, T., M. Hasitz, and G. A. Jamieson. 1978. Platelet glycocalicin: interaction with thrombin and role as thrombin receptor of the platelet surface. $J$. Biol. Chem. 253(10):3435-3443.

13. Bar-Shavit, R., A. Kahn, G. D. Wilner, and J. W. Fenton, II. 1983. Monocyte chemotaxis: stimulation by specific exosite region in thrombin. Science (Wash. DC). 220:728-731.

14. Zimmerman, G. A., T. M. McIntyre, and S. M. Prescott. 1986. Thrombin stimulates neutrophil adherence by an endothelial cell-dependent mechanism. Ann. NY Acad. Sci. 485:349-368.

15. Chen, L. B., and J. M. Buchanan. 1975. Mitogenic activity of blood components. I. Thrombin and prothrombin. Proc. Natl. Acad. Sci. USA. 72:131135.

16. Chen, L. B., N. N. H. Teng, and J. M. Buchanan. 1976. Mitogenicity of thrombin and surface alterations on mouse splenocytes. Exp. Cell Res. 101:4146.

17. Huang, R-S., A. Sorisky, W. R. Church, E. R. Simons, and S. E. Rittenhouse. 1991. "Thrombin" receptor-directed ligand accounts for activation by thrombin of platelet phospholipase $\mathrm{C}$ and accumulation of 3-phosphorylated phosphoinositides. J. Biol. Chem. 266(28):18 435-18 438. 